\title{
POSITIVE OPERATORS ON A BANACH SPACE AND THE FEJÉR-RIESZ THEOREM
}

\author{
A. G. MIAMEE AND H. SALEHI ${ }^{1}$
}

\begin{abstract}
The Fejér and Riesz theorem on the factorization of nonnegative trigonometric polynomials is extended to the nonnegative operator valued trigonometric polynomials on a Banach space. The work is based on the analysis of quasi square roots of nonnegative operator valued functions on a Banach space.
\end{abstract}

1. Introduction. Several authors have generalized the classical theorem of Fejér and Riesz which asserts that every nonnegative trigonometric polynomial $f$ is $|\varphi|^{2}$ where $\varphi$ is an analytic trigonometric polynomial. For finite dimensional matrices the generalization was found by Rosenblatt [3]. Helson [1] proved a version of the Fejér-Riesz theorem for an infinite dimensional matrix valued function whose entries are trigonometric polynomials with degrees bounded by a fixed constant under the assumption that the matrix valued function is factorable. Rosenblum [4] extended Helson's work as follows: Let $f$ be a nonnegative operator valued function on a Hilbert space

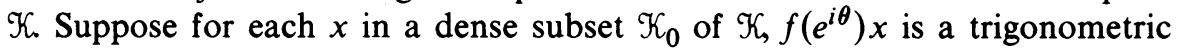
polynomial. Then $f$ is factorable, $f=\Phi^{*} \Phi$, and $\Phi$ can be chosen so that for each $x \in \mathcal{K}_{0}, \Phi\left(e^{i \theta}\right) x$ is an analytic trigonometric polynomial.

The purpose of this note is to extend to the separable Banach space case the main result of Rosenblum. The crucial tool throughout this note is the availability of a quasi square root for a nonnegative operator valued function on a Banach space which we established in [2].

2. Preliminaries. Let $\mathscr{X}$ and $\mathscr{Y}$ be separable Banach spaces. $\mathscr{X}^{*}$ will denote the Banach space of all conjugate linear functionals on $\mathcal{X} B(\mathscr{X}, \mathscr{Y})$ denotes the Banach space of all bounded linear operators on $\mathcal{X}$ into $\mathscr{Y}$. An operator $T$ in $B\left(\mathscr{X}, \mathfrak{X}^{*}\right)$ is called nonnegative if $(T \mathcal{X})(x) \geqslant 0$ for all $x \in \mathfrak{X} . B^{+}\left(\mathfrak{X}, \mathscr{X}^{*}\right)$ stands for the class of all nonnegative operators on $\mathscr{X}$. Let $f=f\left(e^{i \theta}\right)$ be a measurable $B\left(\mathfrak{X}, \mathfrak{X}^{*}\right)$-valued function on $[0,2 \pi]$. $f$ is said to be weakly summable if for each $x, y \in \mathcal{X},\left(f\left(e^{i \theta}\right) x\right)(y)$ is summable, and uniformly summable if $\int_{0}^{2 \pi}\left\|f\left(e^{i \theta}\right)\right\| d \theta<\infty$.

In [2] we proved the following basic result.

2.1. Lemma. Let $f=f\left(e^{i \theta}\right)$ be a weakly summable $B^{+}\left(\mathcal{X}, \mathcal{X}^{*}\right)$-valued function on $[0,2 \pi]$. Then there exists a separable Hilbert space $\mathcal{K}$ and a measurable

Received by the editors April 11, 1974 and, in revised form, December 20, 1974 and February 10, 1975.

AMS (MOS) subject classifications (1970). Primary 42A88, 42A96; Secondary 60G10.

Key words and phrases. Banach space, nonnegative operator valued functions, trigonometric polynomials, invariant subspaces, factorization.

${ }^{1}$ Supported in part by NSF GP-28658.

- American Mathematical Society 1976 
$B(\mathcal{X}, \mathscr{K})$-valued function $Q=Q\left(e^{i \theta}\right)$ such that $f\left(e^{i \theta}\right)=Q^{*}\left(e^{i \theta}\right) Q\left(e^{i \theta}\right) ;$ we call $Q$ a "quasi square root of $f$ ".

Let $\mathcal{K}$ be a Hilbert space. Denote by $L_{2}(\mathcal{K})$ the Hilbert space of all $\mathscr{K}$-valued functions $g=g\left(e^{i \theta}\right)$ such that $\int_{0}^{2 \pi}\left\|g\left(e^{i \theta}\right)\right\|^{2} d \theta<\infty$. The inner product of two functions $g_{1}=g_{1}\left(e^{i \theta}\right)$ and $g_{2}=g_{2}\left(e^{i \theta}\right)$ in $L_{2}(\mathcal{K})$ is given by

$$
\left(g_{1}, g_{2}\right)=\frac{1}{2 \pi} \int_{0}^{2 \pi}\left(g_{1}\left(e^{i \theta}\right), g_{2}\left(e^{i \theta}\right)\right) d \theta .
$$

The operator $U$ defined to be multiplication by $e^{i \theta}$ is unitary and a shift. The subspace $H^{2}(\mathcal{K})$ consisting of those functions $g=g\left(e^{i \theta}\right)$ in $L_{2}(\mathcal{K})$ for which $\int_{0}^{2 \pi} g\left(e^{i \theta}\right) e^{-i n \theta} d \theta=0$ for all $n<0$ is an invariant subspace of $U$. A weakly measurable $B(\mathscr{X}, \mathcal{K})$-valued function $A=A\left(e^{i \theta}\right)$ is said to be analytic if $A x \in H^{2}(\mathcal{K})$ for each $x \in \mathcal{X}$.

Now we give the definition of factorability of a weakly summable nonnegative operator valued function on a Banach space.

2.2. Definition. I Let $f=f\left(e^{i \theta}\right)$ be a weakly summable $B^{+}\left(\mathcal{X}, \mathfrak{X}^{*}\right)$-valued function on $[0,2 \pi]$. We say that $f=f\left(e^{i \theta}\right)$ is factorable if there exists a Hilbert space $\mathcal{K}$ and an analytic $B(\mathcal{X}, \mathcal{K})$-valued function $A=A\left(e^{i \theta}\right)$ such that

$$
f\left(e^{i \theta}\right)=A^{*}\left(e^{i \theta}\right) A\left(e^{i \theta}\right),
$$

in the sense that for each $x$ and $y \in \mathscr{X}$ we have

$$
\left(f\left(e^{i \theta}\right) x\right)(y)=\left(A\left(e^{i \theta}\right) x, A\left(e^{i \theta}\right) y\right) \quad \text { a.e. }
$$

In Definition 2.2 one can show that (see [2])

$$
A\left(e^{i \theta}\right)=\sum_{k=0}^{\infty} A_{k} e^{i k \theta}
$$

where $A_{k}$ 's are bounded operators on $\mathcal{X}$ into $\mathscr{K}_{0}$ In fact there exists a constant $c>0$ such that

$$
\sum_{k=0}^{\infty}\left\|A_{k} x\right\|^{2} \leqslant c\|x\|^{2} \quad \text { for all } x \in \mathcal{X} .
$$

Let $Q$ be as quasi square root of $f$. Let $\Re_{n}(Q)$ denote the smallest subspace of $L_{2}(\mathcal{K})$ invariant with respect to shift $U=e^{i \theta}$ that contains the set $\left\{Q\left(e^{i \theta}\right) e^{i k \theta} x, x \in \mathcal{X}, k \geqslant n\right\}$. Let $\Re_{+\infty}(Q)=\cap_{n \geqslant 0} \Re_{n}(Q)$. We need the following lemma proved in [2].

2.3. Lemma. Suppose $f=f\left(e^{i \theta}\right)$ is a weakly summable $B^{+}\left(\mathcal{X}, \mathcal{X}^{*}\right)$-valued . function on $[0,2 \pi] . f$ is factorable if and only if $\Re_{0}(Q)$ contains no nontrivial reducing subspace of $U$.

2.4. Corollary. A weakly summable $B^{+}\left(\mathcal{X}, \chi^{*}\right)$-valued function $f=f\left(e^{i \theta}\right)$ is factorable if and only if $\Re_{+\infty}(Q)=\{0\}$.

Following Helson [1, p. 121] we introduce the following definition.

2.5. Definition. An analytic $B(\mathscr{X}, \mathcal{K})$-valued function $A$ is called outer if $\Re_{0}(A)=H^{2}(\mathcal{K})$. 
3. Main result. In this section we extend to the separable Banach space case the Fejér-Riesz theorem. Our version of the result reads as follows.

3.1. MAIN THEOREM. Let $f=f\left(e^{i \theta}\right)$ be a $B^{+}\left(\mathcal{X}, \mathscr{X}^{*}\right)$-valued function on $[0,2 \pi]$ which is uniformly summable. Suppose that for each $x$ in a dense subset $\mathfrak{X}_{0}$ of $\mathcal{X}, f\left(e^{i \theta}\right) x$ is a trigonometric polynomial in $\mathcal{X}^{*}$ of degree $\leqslant n(x)<\infty$. Then there exists an outer factor $A$ such that $f\left(e^{i \theta}\right)=A^{*}\left(e^{i \theta}\right) A\left(e^{i \theta}\right)$, a.e. In this case for each $x \in \mathcal{X}_{0}, A\left(e^{i \theta}\right) x$ is an analytic polynomial in $\mathcal{K}$ of degree $\leqslant n(x)$.

Proof. Let $x \in \mathfrak{X}_{0}$ and $k \geqslant 0$. Take $N=n(x)+k$. Then for any polynomial of the form

$$
p\left(e^{i \theta}\right)=\sum_{j=N}^{N+m} e^{i j \theta} Q\left(e^{i \theta}\right) y_{j}, \quad n \geqslant 0,
$$

we have

$$
\begin{aligned}
\left(e^{i k \theta} Q\left(e^{i \theta}\right) x, \sum_{j=N}^{N+M} e^{i j \theta} Q\left(e^{i \theta}\right) y_{j}\right) & =\sum_{j=N}^{N+M}\left(e^{i(k-j) \theta} Q\left(e^{i \theta}\right) x, Q\left(e^{i \theta}\right) y_{j}\right) \\
& =\frac{1}{2 \pi} \sum_{j=N}^{N+M} \int_{0}^{2 \pi} e^{-i(j-k) \theta}\left(f\left(e^{i \theta}\right) x\right)\left(y_{j}\right) d \theta=0,
\end{aligned}
$$

because $j-k \geqslant N-k=n(x)$. Thus $e^{i k \theta} Q x \perp \Re_{N}(Q)$. But $\Re_{N}(Q)$ $\supseteq \mathfrak{\Re}_{+\infty}(Q)$. So we get $e^{i k \theta} Q x \perp \mathfrak{R}_{+\infty}(Q)$, for all $x \in \mathfrak{X}_{0}, k>0$. Now let $x \in \mathcal{X}$ and $k>0$ and let $x_{n} \in \mathcal{X}_{0}$ be such that $x_{n}$ converges to $x$. Since we have

$$
\begin{aligned}
\int_{0}^{2 \pi}\left\|e^{i k \theta} Q x_{n}-e^{i k} Q x\right\|^{2} d \theta & =\int_{0}^{2 \pi}\left\|Q\left(e^{i \theta}\right)\left(x_{n}-x\right)\right\|^{2} d \theta \\
& \leqslant\left\|x_{n}-x\right\|^{2} \int_{0}^{2 \pi}\left\|Q\left(e^{i \theta}\right)\right\|^{2} d \theta
\end{aligned}
$$

we see that $e^{i k \theta} Q x_{\eta}$ converges to $e^{i k \theta} Q x$ in $L_{2}(\mathscr{K})$. Hence for each $k \geqslant 0$ and $x \in \mathcal{X}$ we have $e^{i k \theta} Q x \perp \mathfrak{R}_{+\infty}(Q)$. This implies that $Q P \perp \mathfrak{R}_{+\infty}(Q)$ for each polynomial $p\left(e^{i \theta}\right)=\sum_{k=0}^{n} x_{k} e^{i k \theta}, x_{k} \in \mathcal{X}$. Thus we get $\mathfrak{N}_{0}(Q)$ $\perp \Re_{+\infty}(Q)$ which means $\Re_{+\infty}(Q)=\{0\}$, because $\Re_{+\infty}(Q) \subseteq \pi_{0}(Q)$. Hence by Corollary 2.4, $f$ is factorable in the form $f\left(e^{i \theta}\right)=B^{*}\left(e^{i \theta}\right) B\left(e^{i \theta}\right)$, where $B\left(e^{i \theta}\right)$ is an analytic $B(\mathcal{X}, \mathcal{K})$-valued function. By Lemma 2.3 the invariant subspace $\Re_{0}(B)$ of $H^{2}(\mathcal{K})$ does not contain nontrivial doubly invariant subspaces. Hence by $[1$, p. 121] it has a representation in the form $\Re_{0}(B)=U V H^{2}\left(\mathscr{K}_{1}\right)$, where $U$ is an outer analytic isometry function mapping the Hilbert space $\mathscr{K}_{1}$ onto $\mathscr{T}_{0}(B)$, and $V$ is an inner function in $\mathscr{K}_{1}$. Define $A=V^{*} U^{*} B$. Then $A$ is outer from $x$ into $\mathscr{K}_{1}$ and $f\left(e^{i \theta}\right)$ $=A^{*}\left(e^{i \theta}\right) A\left(e^{i \theta}\right)$. Finally suppose $k \geqslant n(x)$. Then by a similar argument given in the beginning of this paragraph we have $\left(e^{-i k \theta} A x, A p\right)=0$, for each trigonometric polynomial $p$ in $\mathcal{K}_{\text {, i.e. }} e^{-i k \theta} A x \perp A p$ for all polynomials $p$ in $\mathcal{K}_{\text {. }}$ So $e^{-i k \theta} A x \perp H^{2}(\mathcal{K})$, because $A$ is outer. This means that $e^{-i k \theta} A x$ has only negative Fourier coefficients, i.e. $A x$ is a polynomial of degree $\leqslant n(x)$.

3.2. RemarK. To establish his result Rosenblum assumed that $f$ satisfies the condition 


$$
\int_{0}^{2 \pi}\left(f\left(e^{i \theta}\right) g\left(e^{i \theta}\right), g\left(e^{i \theta}\right)\right) d \theta \leqslant c \int_{0}^{2 \pi}\left(g\left(e^{i \theta}\right), g\left(e^{i \theta}\right)\right) d \theta
$$

for each $g$ in $L_{2}(\mathscr{K})$. This condition can be shown to imply the uniform integrability of $f$.

3.3. REMARK. Our original interest in this problem stemmed from the study of Banach space valued stationary stochastic processes. Our theorem has a bearing in the prediction theory of such processes.

\section{REFERENCES}

1. H. Helson, Lectures on invariant subspaces, Academic Press, New York, 1964. MR 30 $\# 1409$.

2. A. G. Miamee and H. Salehi, Factorization of positive operator valued functions on a Banach space, Indiana Univ. Math. J. 24 (1974), 104-113.

3. M. Rosenblatt, A multi-dimensional prediction problem, Ark. Mat. 3 (1958), 407-424. MR 19, 1098.

4. M. Rosenblum, Vectorial Toeplitz operators and the Fejer-Riesz theorem, J. Math. Anal. Appl. 23 (1968), 139-147. MR 37 \#3378.

Department of Mathematics, Arya-Mehr University of Technology, Tehran, Iran

Department of Mathematics, Michigan State University, East Lansing, Michigan 48824 